\title{
Using Digital Image Processing to Make an Intelligent Gate
}

\author{
Sundus K. E. \\ College of Computer Sciences and Mathematics, \\ University of Mosul \\ Mosul, Iraq
}

\begin{abstract}
This paper presents an automatic system for controlling and dominating building gate based on digital image processing. The system begins with a digital camera, which captures a picture for that vehicle which intends to enter the building, then sends the picture to the computer. Image analyses performed to detect and recognize the vehicle, and matching the vehicle's image with the stored database of the permissible vehicles. Then, the computer sends a signal to the electromechanical part that controls gate to open and permits the vehicle to enter the building in case of the vehicle's image matches any image in the database, or sends an apology voice message in case of no identical image.
\end{abstract}

The system is regarded as an empirical and it was applied on various types of vehicles. Results obtained were accurate and the system is successful for all vehicles used in the system test.

Keywords-image processing; color recognition; patch recognition

\section{INTRODUCTION}

Due to the technical difficulties and their complexity in some fields, researchers worked hard to find efficient algorithms that help to find alternative solutions appropriate to reach speedy solution of the problems[1]. Especially in using computer in the field of security and protection, the objects recognition by using computer becomes very necessary for making decisions.

Nowadays, there is a big scientific development in the informatics and programming techniques, in which the images took a very important role in various zone such as medicine media, education, design, industry, security etc [2]. The main reason for this development is the wide using of computer in all these zones. The digital image permits to get images instantly without needing for chemical treatments. The image processing is used in many computer vision applications [3].

It notice that the most researches nowadays, especially image processing researches, tend to use in security field, because the digital images become the main dependent component in most electronic devices used in this field. Where it can store a group of images in a computer database and then using images processing programs to recognize the captured pictures from monitoring camera can make the right decision depending on matching the recognized image with the stored database.

\author{
AL_Mamare S. H. \\ College of Computer Sciences and Mathematics, \\ University of Mosul \\ Mosul, Iraq
}

Due to increasing need for protecting the materials and the articles in the houses, directorates, etc, this paper used a digital images and microcontroller to control opening and closing a building gate.

In the section (III.A) of this paper, there is a declaration and illustration of the program used to recognize the vehicle and the main block diagram for decision making reinforced by figures of recognition stages. While section (III.B) talks about hardware part used as a model to represent the building gate controlled by Arduino. Section (IV) includes an application example. In addition, the conclusions are in section (V).

\section{RELATED WORK}

There are many recent ways to control entering building gates e.g. techniques for recognition such as fingerprint, iris print and face features by using remote control hardware or special identity card. The recognition applications on digital images are developed such as in case of fingerprint recognition or recognition of hidden text in the images[4].

In (2005) Yang proposed two algorithms for security system: the first was a system for humane face recognition and the other was a system for motion detection, the system based on Eigen edge technique [5]. In (2006), Kouma introduced real_time representation for security system based on real time principle to recognize the human face [6]. In (2010), Wael designed real time system to control the security of the building by using distribution mobile technique [7].

\section{The Proposed System}

The proposed system consists of a software part and a hardware part as follows:

\section{A. Software}

This paper used the patch of each vehicle to permit it to enter the building through the gate. The proposed algorithm for recognition (based on recognizing the patch of the vehicle) consists of two main stages: the first stage recognizes the border of the patch, and the second stage recognizes the patch contents.

Fig. 1 illustrates the general diagram of the algorithm, as presented by [8][9] with changes to be adequate with the patch image. 


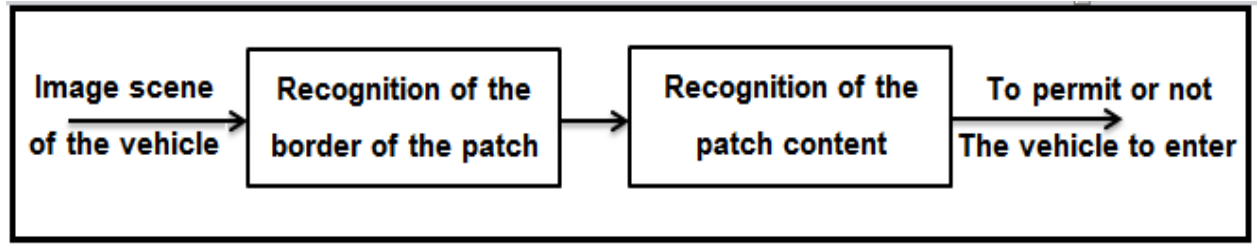

Fig. 1. Block Diagram of Recognition Process.

1) Patch's Border Recognition Steps:

Step1: A captured image enters to the computer and the program read it. Fig. 2 represents a scene for vehicle intended to enter the building.

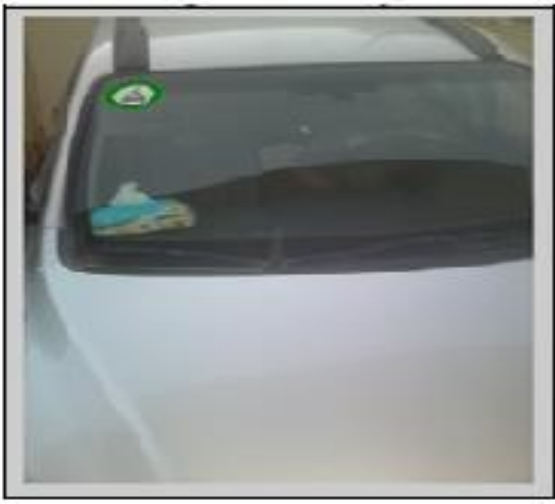

Fig. 2. Scene for vehicle intended to enter the building.

Step2: Apply preprocessing step which involves the following: layers.

a) Reduinge the image size to reduce the execution time.

b) Converting the color image into red, green, and blue

c) Choosing threshold value for each layer by using trial and error method. The threshold used in this research was $(95,130,88)$ for red, green and blue layers respectively for patch border in green color.

d) Converting the previous image into binary image depending on threshold values to ease data process.

e) Removing the noise outside the border of the patch from the produced image, see Fig. 3 .

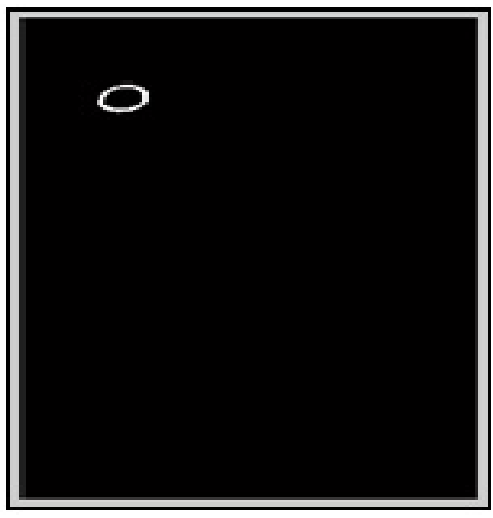

Fig. 3. The image after preprocessing
Step3: Segmentation, in this step the patch segment is clipped from the original colored image by taking the maximum and minimum values of $\mathrm{X}$ and $\mathrm{Y}$ coordinates for the image. See Fig. 4.

Step4: Edge detection for the patch image is applied, see Fig. 5. Then, converting the border shape of the patch into a chain code and checking that the shape is circle, or stopping the processing because the patch is illegal and send an apology voice message.

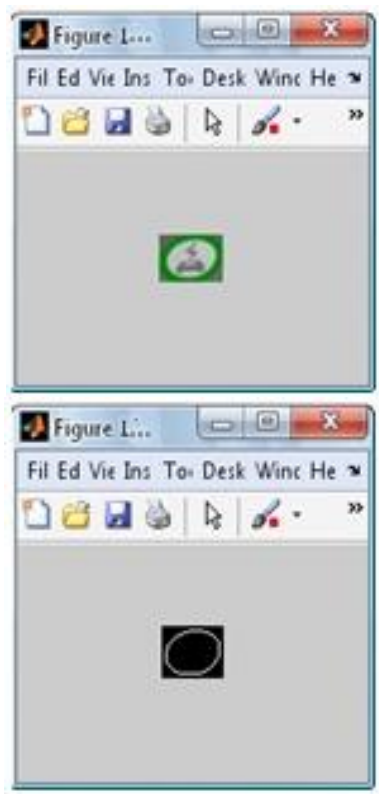

Fig. 4. A clipped image of patch

Fig. 5. The border of patch

2) Recognizing The Inside Contents Of The Patch This involves the following steps:

Step1: Converting the clipped segment of the colored image into a binary image.

Step2: Removing the border of the patch's image from the image, see Fig. 6.

Step3: Appling edge detection on the objects inside the patch by using chain code. In this step, the recognition depends on a number of objects included the patch's image and the chain code to achieve the objective.

Step4: Matching between the chain code of each object with the database to recognize the vehicle is permissible to enter or not. 


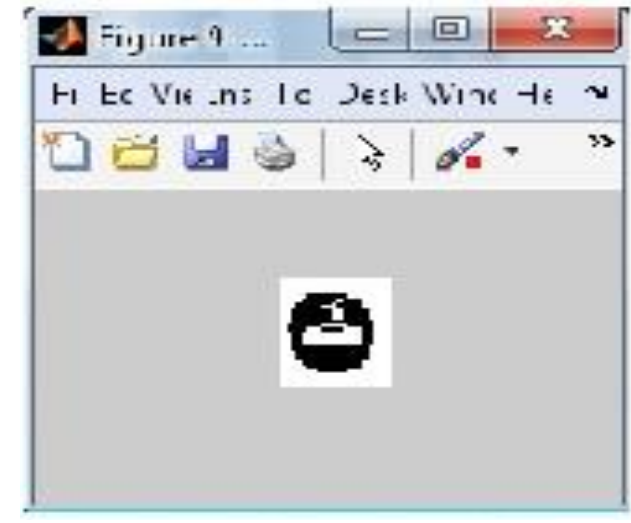

Fig. 6. The inside patch contents.

\section{B. Hardware Components}

They include of the following parts:

\section{1) Simulated Model}

A small model used to simulate the real building. The model $1 * 1 \mathrm{~m} 2$ area represents $10 * 10 \mathrm{~m} 2$ real building i.e. the scale is $1: 10$ the height of the model is $28.5 \mathrm{~cm}$, the slide gate is $20 \mathrm{~cm}$ high and 28.5 width. See Fig. 7. Small electronic motor uses $(12 \mathrm{~V})$ to move the gate by arm joining the motor with gate. Fig. 8 illustrates the mechanical components fixed to the model gate.

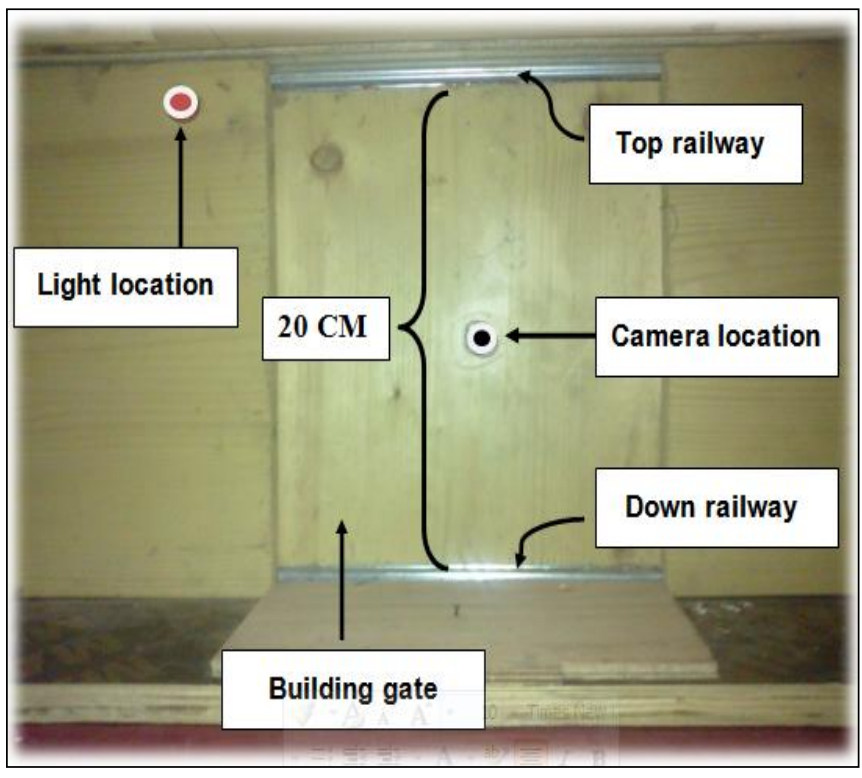

Fig. 7. Front view of the gate.

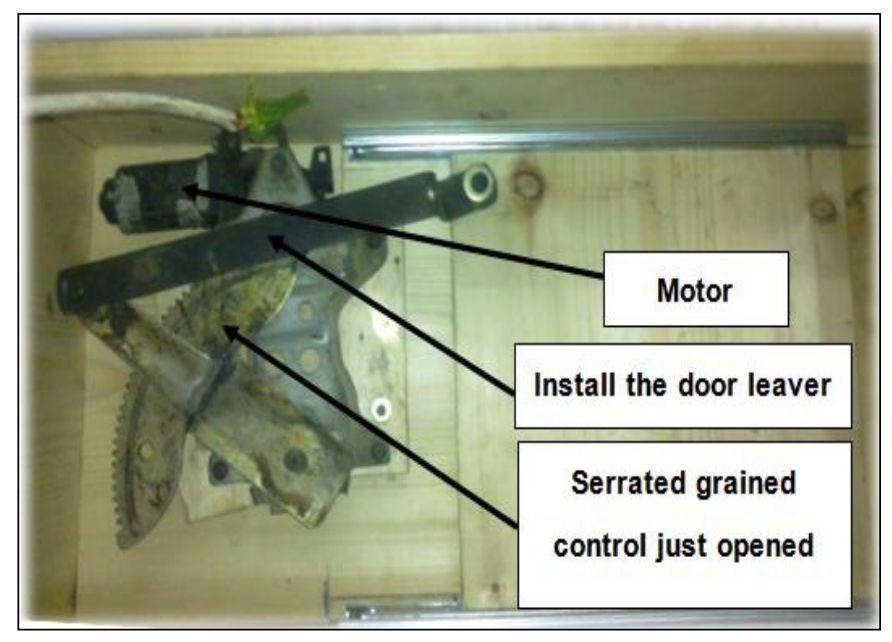

Fig. 8. Inside view of the gate

\section{2) Arduino board}

The Arduino board is a small microcontroller board. The Arduino board can write programs and create interface circuits to read switches and other sensors [10]. Arduino UNO is used in this paper.

\section{3) driver}

Driver is the join between the motor and the Arduino UNO. It consists of: relay, diode, copper pin, headers 18 pin, board, and capacitor, see Fig. 9.

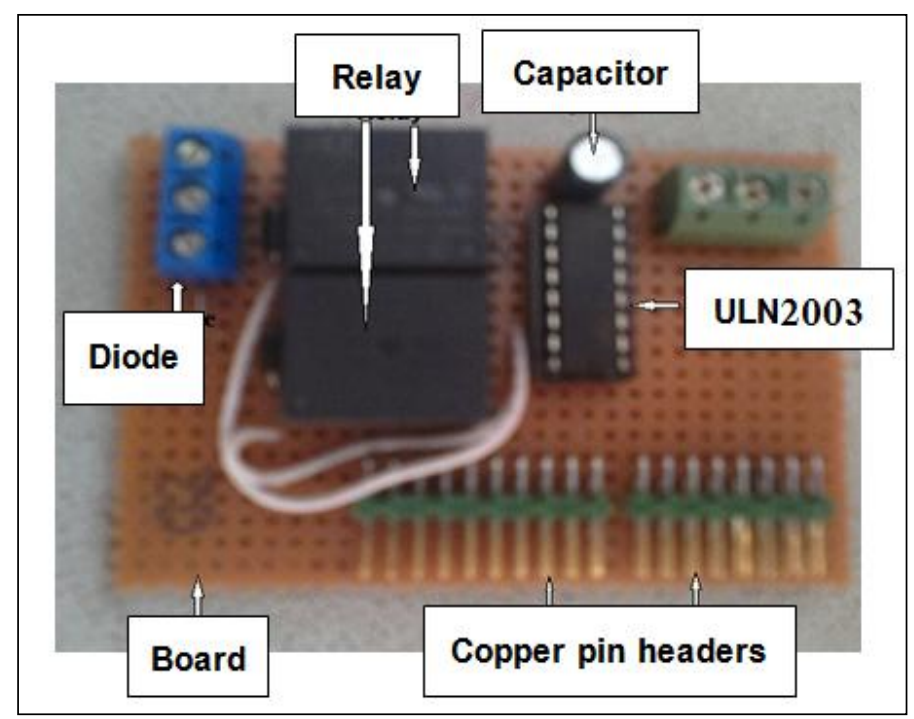

Fig. 9. Drivers Components 


\section{4) Electronic Circuit}

The electronic circuit used in the model of proposed system to open, close, and stop the building gate is illustrated in Fig.10.

Fig. 11 shows the flowchart of the system procedure.

The system procedure begins with a picture captured by a digital camera for the vehicle that stopped before the building gate intending to enter the building. The camera sends a captured picture to the computer. The computer recognition program runs to recognize the vehicle depending on the color and shape of the patch and matching it with sorted database. In case of the matching occurred, the system will permit the vehicle to enter, by sending a signal (means open) to the electromechanical part (which is fixed to the gate to control opening and closing the gate), then the gate will open.

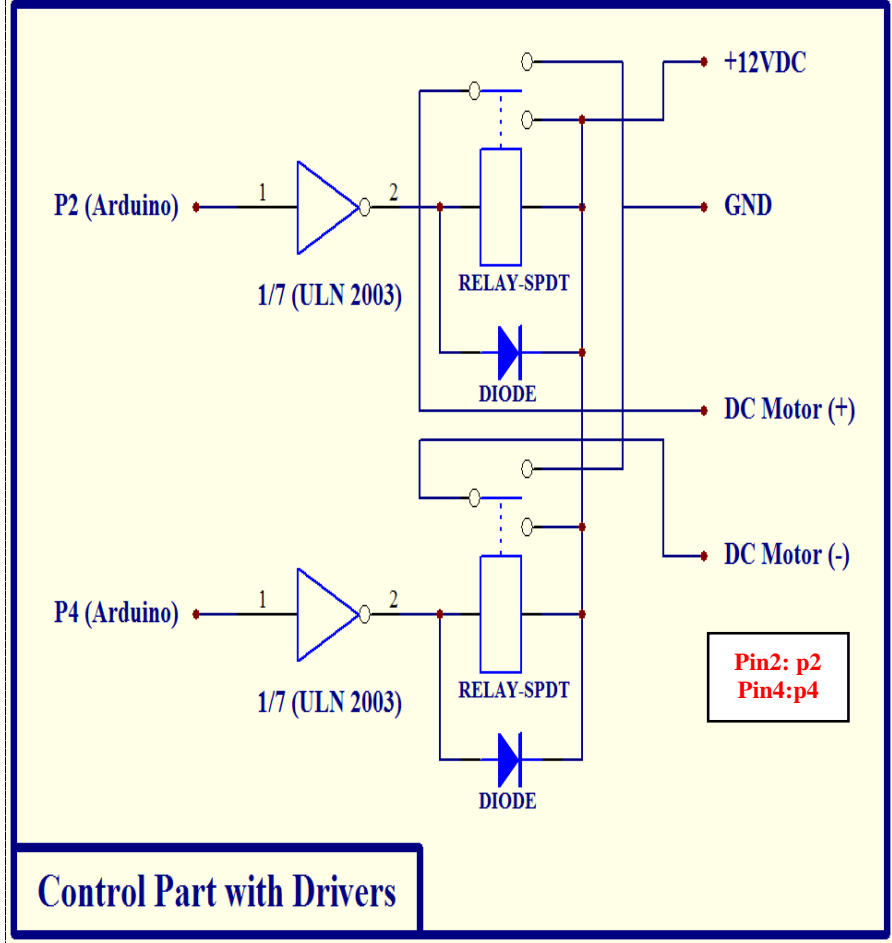

Fig. 10. Electronic circuit Hardware parts. The system procedure

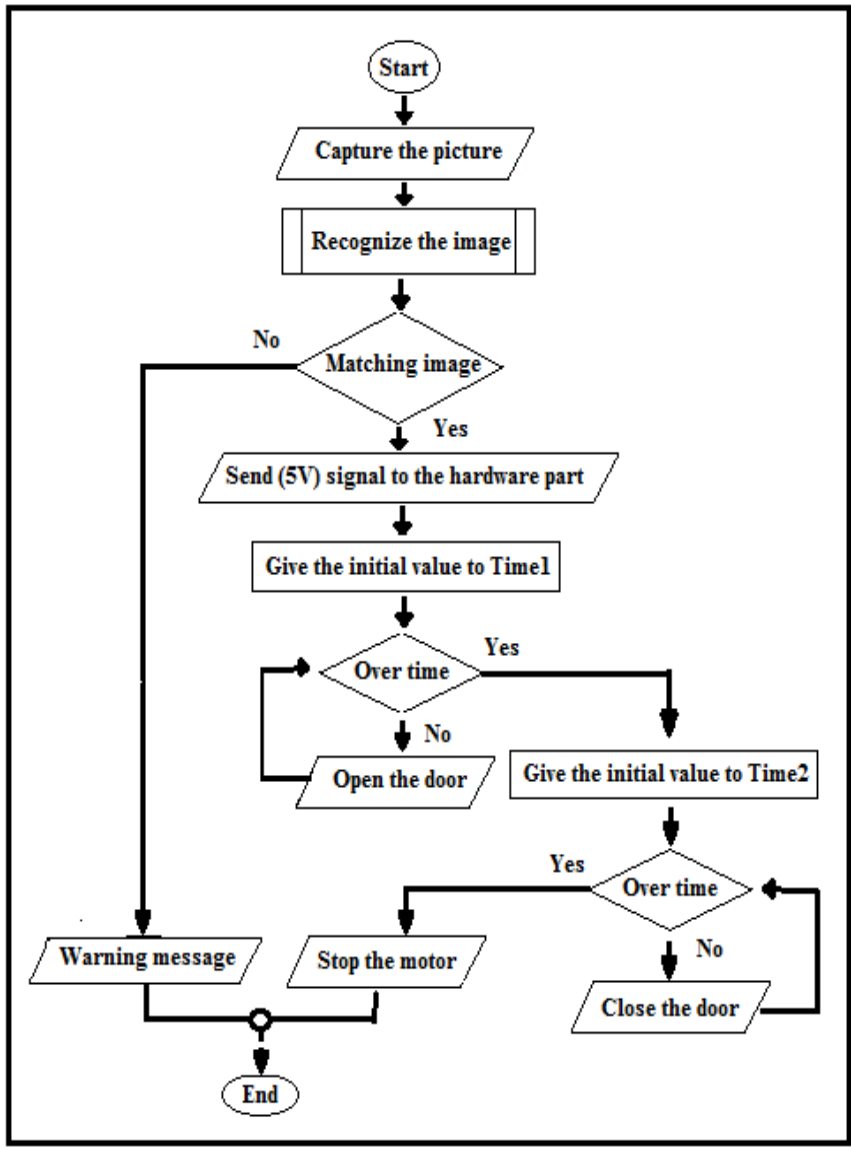

Fig. 11. The flowchart of the system procedure

While in case of the no matching occurs the system will send an apology voice message (means that the vehicle is not permissible to enter), and the system electronic circuit works as follows:

Opening the gate : In case of that the patch of the vehicle matches one in database, the computer will sends a signal $(5 \mathrm{~V})$ to pin2 in the Arduino board which in its role sends a signal(5V) to the ULN2003 in the driver board, the driver sends $(5 \mathrm{~V})$ to the fist relay, the first relay sends $(12 \mathrm{~V})$ to the motor which will work to open the gate after getting the current. See Fig. 10. 
Closing the gate: When the time of entering the vehicle to the building through the gate is out, the segment of code that related to closing the gate will be executed in the computer and sends a $(5 \mathrm{~V})$ signal to the Arduino board which in its role sends a signal to the ULN2003 in the driver board.The ULN2003 sends a signal to the second relay. The second relay sends $(12 \mathrm{~V})$ to the motor which begins to move to close the gate by switching the polarity of the (12V). See Fig. 10.

Stopping the motor: When opening time is out, the segment of code related to stop the motor will be execute in the computer and sends a $(0 \mathrm{~V})$ signal to in the Arduino board which in its role sends these signals to the ULN2003 which sends signals to the first and second relays which in their role stop the motor current and the motor will stop, see Fig. 10.

\section{EXAMPLE}

The proposed algorithm of recognition applied on various types of real vehicles and the sample of dummy vehicle used to apply the simulated model to open and close the gate. See Fig. $12-14$, scene for the gate to permit the vehicle to enter into the building.

\section{CONCLUSIONS}

Automatic controlling and domination on the building gates are regarded as an important matter nowadays whether for vehicles or persons, that's due to the difficulty of the traditional systems because they are not accurate and required too much time, effort and cost. Even recent systems need for fixing special sensors in the building or needs mobile network connection to work. While the hardware and software in this proposed system are simple, inexpensive and available.

The matter of using computer to control and dominate the gate systems became very urgent to arrange, manage and control the security of the buildings. Thus, the proposed system is a computer system based on digital image processing and pattern recognition techniques. This system invests these techniques in the security field through recognizing the patch of the vehicle, which intends to enter the building. The results show $95 \%$ recognition percentage for the used samples of the real passenger's cars and the simulated dummy vehicles.

To increase security of system, it is recommended for future works to add recognition of license plate of vehicle in addition to patch recognition, which will increase the system authentication and reliability that mean increasing the efficiency of security work.

\section{ACKNOWLEDGMENT}

Our thanks to the journal staff for their patience and cooperation.

\section{REFERENCES}

[1] E. Abbas, H. Farhan, "Face Recognition using DWT with HMM", Eng. \& Tech. Journal, vol.30, No.1, 2012, pp 142-154.

[2] M. Ahmed, A. Barzan, "Automatic Features Recognition for Symmetrical Shapes", Eng. \& Tech. Journal, vol.30, No.12, 2012, pp 2027-2043

[3] L. Crowley, H. Daniel and R. Emonet, " Autonomic Computer vision system", In International Conference on computer vision system, Icvs, vol. 17, 2007.

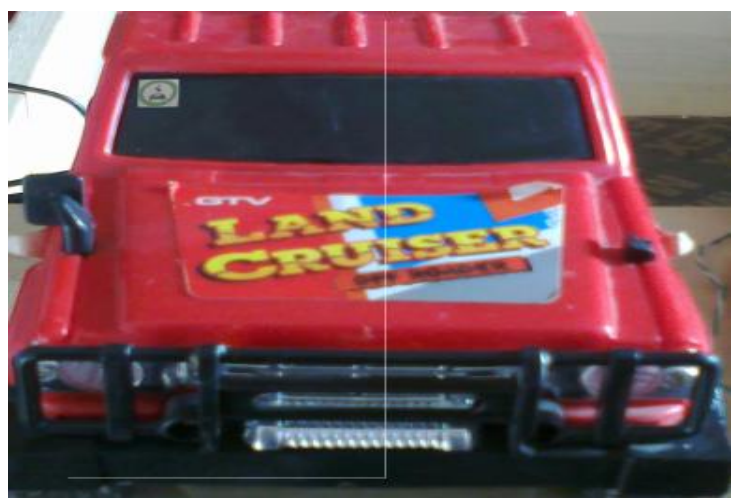

Fig. 12. Captured picture

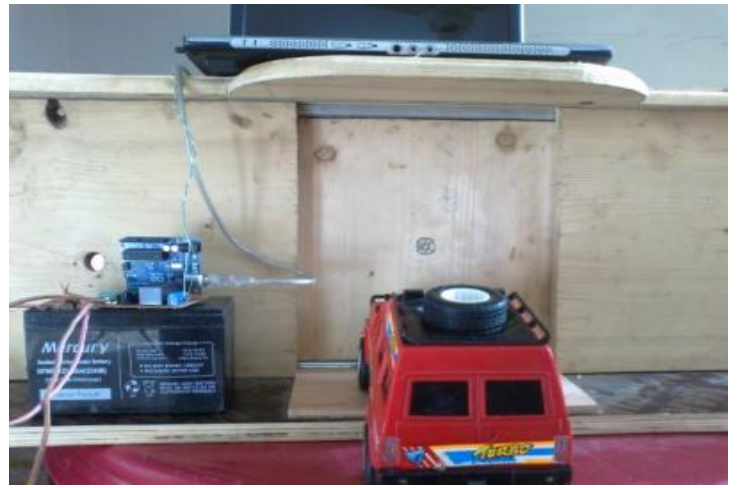

Fig. 13. Scene before opening the gate

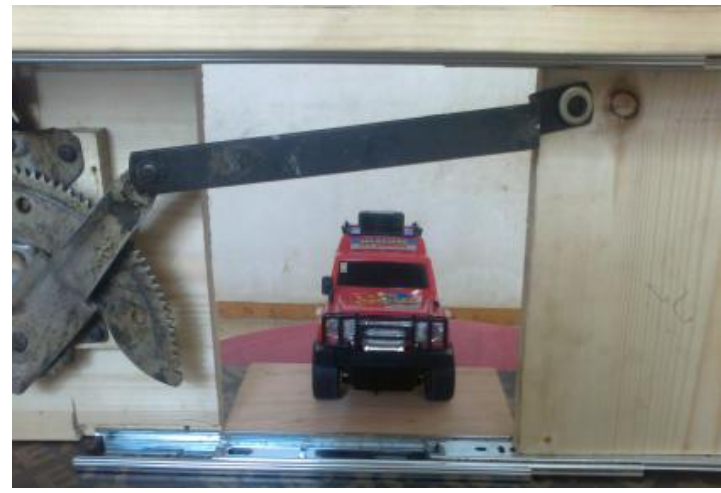

Fig. 14. Scene after opening the gate from inside the building model

[4] M. Kadum, "Detection of Hidden Object In Speech Based on Fast Fourier Transform Algorithm", Eng. \& Tech. Journal, vol.29, No.2, 2011, pp 375-385.

[5] J. Yang, “ Algorithms For An Integrated Vision-Based Home Security System”, Multimedia University, Malaysia , 2005.

[6] J. Kouma, "Intelligent Home Security System", Applied Physics and Electronics, SE-901 87, Umea university, Sweden, 2006.

[7] W. Wael, "Design And Implementation Real Time System For Building Security By Using Distributed Mobile Technique Method", MSc. thesis, Computer Sciences and Mathematics, University of Mosul, 2010.

[8] R. Jasim, S. Khaleel, " Traffic Sign Recognition using Chain Code", AL-Rafiden Journal of Computer Sciences and Mathematics, No.9, vol.2, 2012.

[9] R. Jasim, S. Khaleel, "Recognition using ChainCode", Iraqi Journal of Statistical Sciences, No. 20, vol.11, 2011, pp 691-713.

[10] M. Banzi , Getting Started with Arduino, $2^{\text {nd }}$ Edition,Copyright, CMassimo Banzi. All rights reservedPrinted in the U.S.A, 2011. 